\title{
Combining Problem Frames and UML in the Description of Software Requirements
}

\author{
Luigi Lavazza $^{1,2}$ and Vieri Del Bianco ${ }^{2}$ \\ ${ }^{1}$ Università dell'Insubria, Dipartimento di Informatica e Comunicazione, \\ Via Mazzini 5, Varese 21100, Italy \\ ${ }^{2}$ CEFRIEL, \\ Via Fucini 2, Milano 20100, Italy \\ \{lavazza, delbianc\}@eefriel.it
}

\begin{abstract}
Problem frames are a sound and convenient approach to requirements modeling. Nevertheless, they are far less popular than other less rigorous approaches. One reason is that they employ a notation that is neither very appealing nor easy to use. The problem frames notation is sufficiently different from other development languages -especially UML- to create an "impedance mismatch": using problem frames to describe requirements does not help the transition to the design phase, makes it difficult for programmers to fully comprehend requirements, and does not favor traceability. As a consequence, problem frames are rarely adopted in software development processes employing UML as a design language. UML itself provides a linguistic support for requirements modeling, which however suffers from several limitations, especially as far as precision and formality are concerned.

The goal of this paper is to combine problem frames and UML in order to both improving the linguistic support for problem frames -while preserving the underlying concepts- and to improve the UML development practice by introducing the problem frames approach, making it seamlessly applicable in the context of the familiar UML language.
\end{abstract}

\section{Introduction}

The Problem Frames approach [1] has the potential to dramatically improve the early lifecycle phases of software projects. Problem frames (PFs) drive developers to understand and describe the problem to be solved, which is crucial for a successful development process.

Nevertheless, PF have some limitations that hinder their application in industrial software development processes. In particular, they are not provided with an adequate linguistic support. For instance, by looking at the PF represented in Fig. 1 it is not immediate to see the association of phenomena with domains, or to see which domain controls which phenomena. Moreover, sometimes it is difficult to represent the nature of shared phenomena; e.g., when they involve complex data structures that are much better modeled via classes.

PFs are not equipped with a unique and clear way for expressing requirements: the modeler has to choose a suitable logic language to predicate about phenomena. 


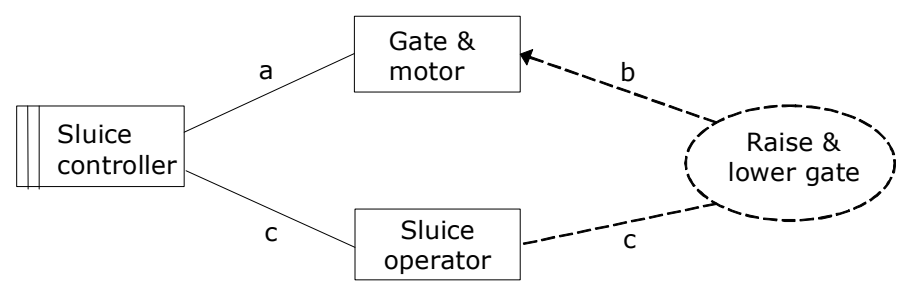
a: SC! $\{$ Clockw, Anti, On, Off $\}$ GM! $\{$ Top, Bottom $\}$

b: GM!\{Open, Shut, Rising, Falling\}

c: SO! Raise, Lower, Stop\}

Fig. 1. A commanded behavior frame: the sluice gate control

Moreover, the PF model causes a sort of "impedance mismatch" with respect to the languages employed in the subsequent development phases. In particular, considering object-oriented developments using UML [4] as a modeling language, it is not immediate to transform PF diagrams and predicates into UML models. Even worse, people involved in a UML-based development that are not used to the PF notation can find it difficult to understand requirements. It is well known that design and implementation are more often successful if developers fully understand the requirements; but a Java programmer that works on the basis of a UML design built according to PFs can find it difficult to read the requirements.

Problem frames require a linguistic support that is easy to use, that allows the relevant information to be represented in a natural and readable way, and that allows a smooth transition to the subsequent development phases. In particular, here we consider development activities based on the usage of UML.

For requirements modeling, UML provides the "use case" diagrams [16]. Unfortunately, use cases suffer from several limitations, amply described in the literature [17], [18]. The main limitations with use case diagrams are that they are neither formal nor rigorous-requirements being described mainly by the text that illustrates the "courses of action"- and they are not intrinsically object-oriented, thus it is not easy to move from use cases to the object-oriented models required by the following phases of development. UML-based development needs to be supported by a technique for requirements modeling that overcomes the limitations of use cases.

We propose to integrate PF and UML: PFs are represented by means of UML, so that they can be used (also) in UML-based development. This integration provides several benefits: on one hand it equips PFs with a popular and easy to use notation. Under this respect our contribution is quite similar to other initiatives aiming at providing UML-based access to requirements modeling methods (e.g., KAOS [6]). In fact, a method can be truly successful only if a large number of professionals are sufficiently convinced of its potential to use it in industrial settings. Using UML to support requirements engineering with PF may help achieve this end.

On the other hand, the usage of UML both as the notation underlying PFs and as a design language smoothes the transition from the requirement elicitation and modeling phase to the design phase -thus facilitating the comprehension of requirements by the developers- and it eases the iteration between problem and solution domains. Moreover, it makes easier to represent traceability relations, since requirements and elements of the solution are represented in a homogeneous way. 
The paper is organized as follows: Section 2 illustrates how problem frames can be defined using UML-based notations. Section 3 tests the ability of the proposed approach to deal with the representation of a couple of concerns that often occur in requirements. Section 4 briefly accounts for related work, while Section 5 draws some conclusions.

\section{Problem Frames with UML}

The most obvious choice for representing requirements in a way that eases UMLbased development is to employ class diagrams (and possibly object diagrams) to represent the structure of the system, and statecharts and OCL statements to specify the behavior of the system [5]. The recently released UML 2.0 [4] features an improved ability to model the structure of systems. In fact, it introduces "composite structures", that allow the modeler to hierarchically decompose a class into an internal structure. The relations among the parts can be specified by means of associations and interfaces.

Unfortunately, OCL [19] is limited with respect to the possibility of specifying temporal aspects: only invariant properties can be formalized, which at most include references to attribute values before or after method execution. It is not possible to reference different time instants in a single OCL formula; namely it is not possible to reference the time distance between events. Therefore, several kinds of important temporal properties of systems cannot be adequately specified.

In order to overcome the aforementioned limitations, Object Temporal Logic (OTL) was defined as a temporal logic extension to OCL [3]. However, OTL extensions do not require to change the OCL metamodel: they can be considered the minimum enhancements of OCL required to deal with time.

OTL formulas are evaluated with respect to an implicit current time instant. In fact, OTL introduces a new primitive as a method of class Time: method eval receives an OclExpression as the parameter $(\mathrm{p})$ and returns the (boolean) value of $\mathrm{p}$ at time $t$. This is denoted as t.eval (p) or, more concisely, as pat. All other typical temporal operators -like Always, Sometimes, Until, etc.- are defined based on method eval. In addition, OTL allows the modeler to reason about time in a quantitative fashion. Properties can be expressed on (possibly infinite) collections of objects of class Time, i.e., on time intervals.

In the rest of the paper OTL is used to express time-dependent properties. Of course, a modeler could express properties and requirements informally, by means of comments associated with model elements. This practice - which is in line with the way UML is generally used in industry- is often acceptable. Nevertheless, here we employ OTL extensively, in order to show that the proposed approach can lead to very precise and rigorous specifications.

\subsection{Problem Frames with UML and OTL}

Problem frames are represented by means of UML diagrams and OTL statements according to the following rules. 
Domains (including the machine) are represented by means of components. This seems a reasonable choice, since components can include any of the properties that characterize domains. In particular it is possible to structure domains into subdomains, while the machine is treated as a sort of "black box component", whose internal details are not given. Different kinds of domains can be represented by means of different kinds of components: we use UML stereotypes to specialize components into causal domains, biddable domains, machines, etc.

Components hide their internal details from the outer world by means of ports. Shared phenomena are represented by means of interfaces, the construct provided by UML to explicitly exchange information among components. In particular, phenomena that are shared between domains D1 and D2 require that components representing D1 and D2 are suitably connected, i.e., they must be equipped with ports and compatible interfaces. Shared phenomena define the interfaces, i.e., they define the operations belonging to the interfaces. Parameters of phenomena become parameters of the operations associated with the interfaces. The details of the domains can be defined in terms of classes or sub-components.

For instance, the required behavior frame (Fig. 2) can be represented by the UML model illustrated by Fig. 3 .

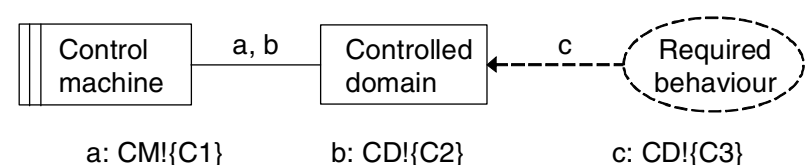

Fig. 2. The required behavior frame

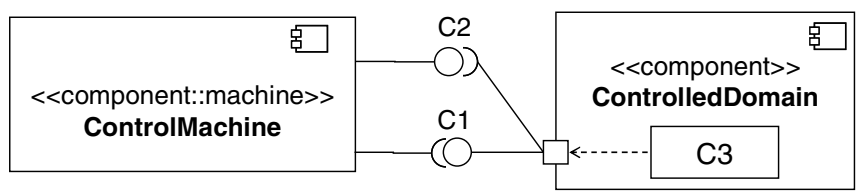

Fig. 3. UML representation of the required behavior frame

Properties are simply expressed as OTL statements involving the proper interface operations and component properties (attributes, states, etc.). Properties of the controlled domain represented in Fig. 3 would be described by OTL statements concerning the context of the ControlledDomain. Alternatively, the behavior of the controlled domain can be expressed by means of statecharts. The requirements for the system are expressed similarly by OTL statements concerning the context of ControlledDomain: OTL can be used to describe the required behavior of $\mathrm{C} 3$.

\subsection{A Commanded Behavior Frame: The Sluice Gate Control}

In order to test the applicability of the proposed approach, in this section we represent the sluice gate control problem by means of UML and OTL.

The problem is defined as follows [1]. A small sluice, with a rising and a falling gate, is used in a simple irrigation system. A computer system is needed to raise and 
lower the sluice gate in response to the commands of an operator. The gate is opened and closed by rotating vertical screws. The screws are driven by a small motor, which can be controlled by clockwise, anticlockwise, on and off pulses. There are sensors at the top and bottom of the gate travel; at the top it is fully open, at the bottom it is fully shut. The connection to the computer consists of four pulse lines for motor control, two status lines for gate sensors, and a status line for each class of operator command. The PF diagram for the sluice gate problem is reported in Fig. 1.

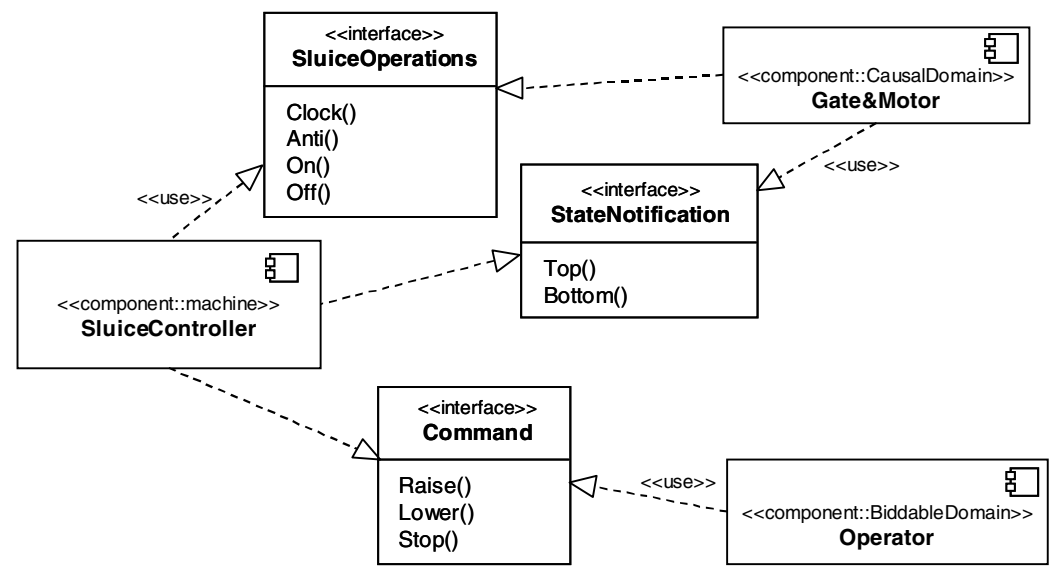

Fig. 4. Sluice gate control: component diagram

The sluice control problem is represented according to our proposal by the class diagram reported in Fig. 4 and by the component diagram reported in Fig. 5. Note that for the sake of clarity, instead of using the "lollypop" notation, in Fig. 4 and Fig. 5 we described the interfaces explicitly, reporting the operations that can be invoked through every interface. It can be noticed that the diagrams incorporate a few choices that improve the clarity of the representation:

- The Gate\&Motor domain is decomposed into the Gate and Motor subdomains.

- The events from the Gate\&Motor are divided into two sets. The messages concerning the state of the gate are emitted by the Gate subdomain, while those concerning the control of the motor are delivered to the Motor subdomain.

- The Gate and Motor subdomains are connected via the GateMotor interface, in order to represent the effect of the motor on the gate.

The behavior of the controlled domain can be described by the OTL statements reported below. Note that for space reasons we do not give the complete specification, but only a subset that is representative of the capabilities of the proposed approach.

The specifications reported below apply only when the gate and motor are working correctly. If it were required to handle failures, then the combined behavior of the two subdomains would be modeled differently.

- The motor is on, if and only iff an on command arrived, and since then no off command arrived: 


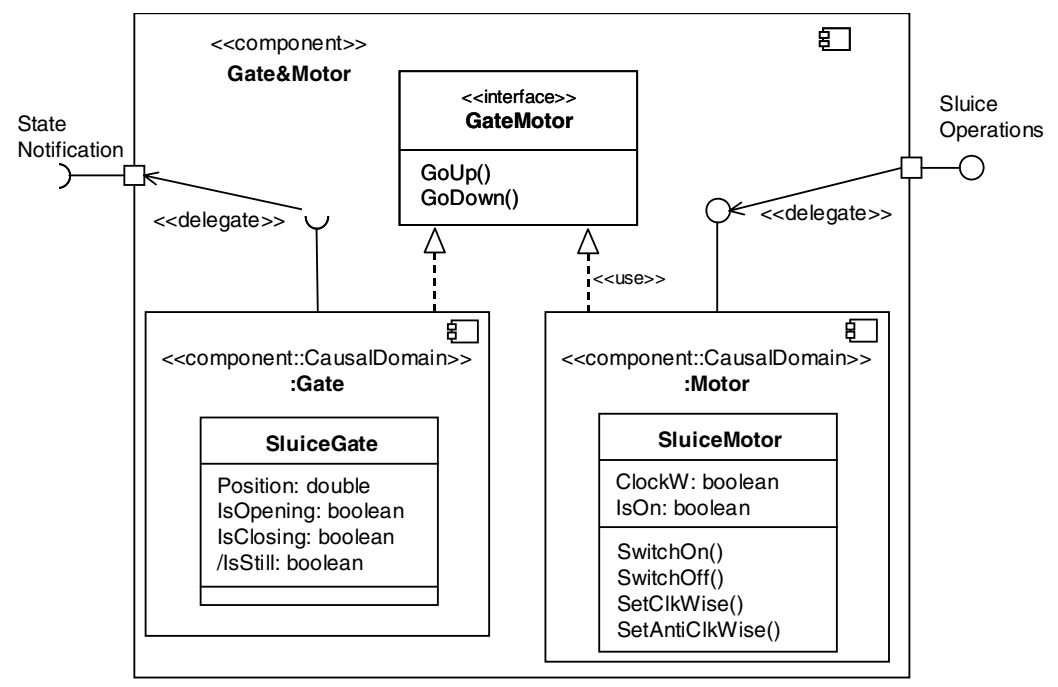

Fig. 5. Sluice gate control: white-box view of the Gate\&Motor component

context Gate\&Motor inv:

SluiceMotor.IsOn = Since $($ not SluiceOperations^off, SluiceOperations^on)

Since $(p, q)$ states that $q$ occurred in the past, and since then $p$ is true.

- The gate is still whenever it is neither opening nor closing: context Sluicegate inv:

self.IsStill= not(self.IsOpeneing or self.IsClosing)

- When the motor is on and moving clockwise the gate is opening: context Gate\&Motor inv:

(SluiceMotor.IsOn and SluiceMotor. ClockW) =

SluiceGate. IsOpening

- When the motor is off the gate is still: context Gate\&Motor inv:

SluiceMotor.IsOff = SluiceGate.IsStill

- When the gate is opening and the position of the gate reaches the top, the Open notification is sent via StateNotification interface port within RT time units, $\mathrm{RT}$ being the allowed reaction time.

context GatesMotor inv:

(SluiceGate.IsOpening and becomes (SluiceGate.position=top))

= WithinF (StateNotification^Open, RT)

The meaning of $\operatorname{WithinF}(p, d)$ is that $p$ will hold within $d$ time units after the evaluation time.

- When the gate is opening the position increases at a constant speed (and similarly the position decreases when the gate is closing):

context sluicegate inv:

Lasted (self.Isopening, D) =

self $\cdot$ position $=$ self $\cdot$ position $($ now $-D)+D^{*}$ speed

The meaning of Lasted $(p, d)$ is that $p$ held for $d$ time units in the past. 
- When the gate is still the position is constant:

context SluiceGate inv:

Lasted ( self. IsStill, D) = (self .position=self .position@ (now-D) )

- The correspondence between operations of the interfaces and methods provided by classes or subcomponents can also be specified by means of OTL. For instance, the following statements specify that the clock and on commands that reach the Gate\&Motor domain are delegated to the SetClkWise and Switchon methods of SluiceMotor respectively.

context GatesMotor inv:

SluiceOperations^Clock = SluiceMotor^SetClkWise context Gate\&Motor inv:

SluiceOperations^ ${ }^{\wedge}$ On $=$ SluiceMotor^Switchon

Note that by the Element^Message notation we denote both the events of sending and receiving a message.

- Additional trivial statements - not reported here- are needed to enumerate the possible states for the gate and motor, to enforce mutual exclusion of states, to specify the initial states of the gate and motor, etc.

It is interesting to note that the behavior of the combination of the given subdomains was modeled quite naturally by establishing correspondences between the states of the gate and the motor, since the gate is affected by the state of the motor (e.g., when the motor is on clockwise the gate is opening). Otherwise, modeling the behavior of the gate in terms of the events that are directed to the motor would not have been as easy, since the gate is not directly affected by the signals that are sent to the motor. In these situations, the availability of OTL eases the description of the continuous behavior of a domain. Specifying that a domain continuously affects another domain would be very hard using only statecharts.

The behavior of the Operator is not specified, i.e., he/she could generate any sequence of commands. It is the job of the sluice controller to guarantee a reasonable behavior for any possible sequence of commands. Here we assume that the requirements for the sluice gate controller are quite simple:

- The Raise and Lower commands from the operator are simply transformed by the machine in pairs of commands (<ClockWise, On $>$ and $<$ AnticlockWise, on $>$ respectively), which are sent to the Motor, unless the Gate is not already Open or Shut, respectively. This behavior is modeled (for the Raise command) by the following OTL code, where MD is the time taken by the motor to react to commands, and ST is a very short time.

context Operator inv:

(Command^Raise and not SluiceGate.position=Top) =

(SluiceOperations ${ }^{\wedge} \mathrm{Clock}$ and SluiceOperations`年)

context Gate\&Motor inv:

(SluiceMotor^Switchon and

WithinP(SluiceMotor^SetClkWise, ST) or

SluiceMotor^SetClkWise and

WithinP (SluiceMotor^Switchon, ST))

and not WithinP(SluiceMotor^SetAnticlkWise, ST)

and not WithinP(SluiceMotor^Switchoff, ST))

implies WithinF(SluiceMotor.IsOn and SluiceMotor.ClockW,MD) 
Note that last statement takes into consideration the possibility that Switchon and SetClkWise do not arrive at the same time, but (quite realistically) separated by a small interval $(\leq S T)$. The statement specifies that if the on and SetClkWise commands arrive (in any order) in a time interval ST and no counter order arrives in the same interval, then the motor will be on in clockwise direction within MD time units.

- The Top and Bottom notifications from the gate, as well as the Stop command from the operator are transformed by the machine into the command off, in order to stop the Motor.

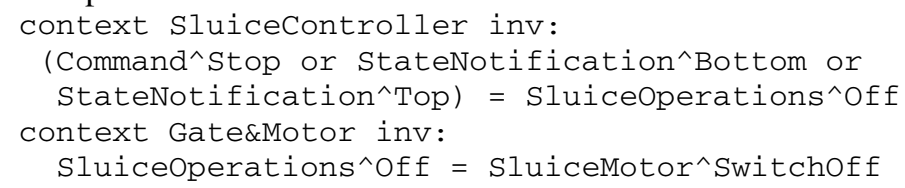

As already mentioned, we could express more complex requirements. For instance, the controller, instead of just "translating" the commands from the operator, as specified above, could ignore any command that is not separated from the previous one by a minimum interval D. This requirement for command Raise can be expressed as follows:

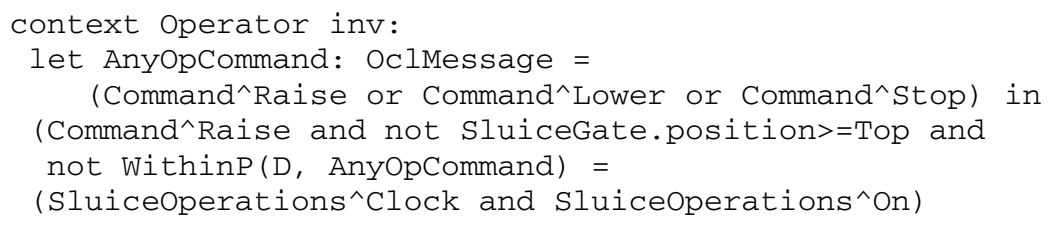

\section{Other Issues}

In order to further test the applicability of the approach, in this section we consider two of the concerns described in [1]. The concerns are illustrated by means of the same examples reported in [1].

\subsection{The Reliability Concern}

Several real-life problems can be more easily understood and described if they are decomposed into subproblems. For example, it is often advisable to deal with the reliability concern in a separate subproblem. Subproblems are best identified as projections of the original problems, i.e., they are obtained by considering the subsets of elements and phenomena that are relevant to the subproblem [1].

Reliability is one of the most common subproblems, since often it is necessary to describe the main problem, and then to take into account reliability issues. In the case of the sluice gate controller, it is quite clear that the specifications given in Section 2.2 apply only when the gate and motor work correctly. However, in general it would be desirable that the sluice gate controller guarantees a reasonable behavior of the motor and gate system even in presence of failures. For instance, if debris are jammed in the gate, preventing it from closing, or if a sensor sticks on or off, the controller should not insist in trying to close the gate. 
A way to tackle the problem -proposed in [1]- consists in observing the system, in order to provide the operator with a stop warning whenever there is the perception that something is going wrong. This "auditing" subproblem is described in Fig. 6, where the auditing machine is in charge of monitoring the system, represented by the Gatesmotor+Controller domain, which includes both the controlling machine (the Sluice controller of Fig. 1) and the controlled domain (the Gate\&motor of Fig. 1). The Audit machine receives all the relevant events generated by the union of the Gate\&motor and the Sluice controller and reacts sending a Stop warning to the safety operator when appropriate. The Safety operator (who may be the same person as the sluice operator) is a biddable domain: he/she should be instructed to issue a stop command whenever a stop warning is received.

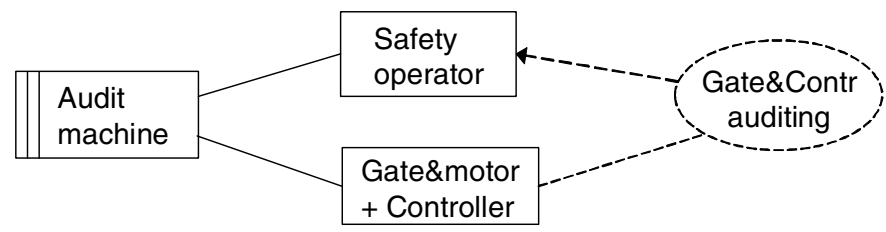

d: AM!\{Stop\} $\quad$ e: GC!\{ClockW, Anti, On, Off, Top, Bottom\}

Fig. 6. Sluice gate controller: auditing reliability

Fig. 6 is a projection of the whole sluice gate control problem, and it is an information display frame. It is therefore interesting to analyse if the UML-based representation of PFs can manage the representation of a projection of the problem, in the form of an information display frame. It is also interesting to consider how the two resulting sets of UML diagrams can be composed.

The required projection of the system is represented in Fig. 7. The collaboration diagram of Fig. 7 is a rather straightforward representation of Fig. 6. The Alarm interface between the AuditMachine and the Safetyoperator consists just of the warning issued by the machine, to which the operator should react by means of a Stop command. Between the AuditMachine and the Gate\&Motor\&Controller the Command\&State interface notifies the AuditMachine of the ClockW, Anti, On, off commands and Top and Bottom states, so that the machine can detect abnormal behaviors of the gate and motor in reaction to commands. The refinement of the diagram reported in Fig. 7 is omitted for simplicity.

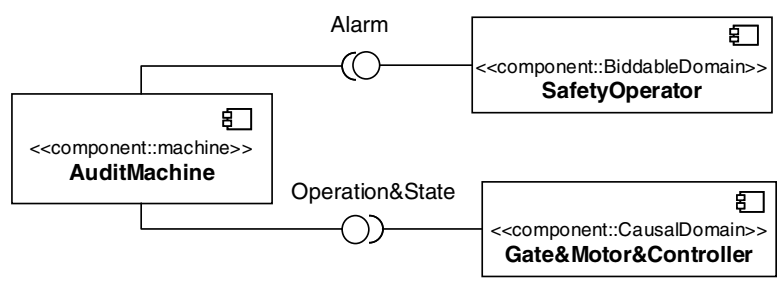

Fig. 7. Sluice gate auditing: component diagram 
The requirements for the auditing system can vary widely, depending on the failures considered. Here we consider a failure detection technique based on the assumption that a failure occurs when the gate fails to react to a command in a given interval.

For instance, the following OTL statement specifies the detection of a failure concerning the lowering of the gate. If the condition for starting lowering the gate (i.e., the motor received the <AnticlockWise, on>commands) was verified $D$ time units ago, and $\mathrm{D}$ is big enough to allow the completion of the operation ( $\mathrm{CT}$ being the expected completion time and MD the motor reaction time), and no counter-order was received, and the gate sensor did not notify the completion of the operation (Bottom signal) then a StopWarning will be issued within one time unit. In the following OTL statement the interface names reported in Fig. 8 are used.

context AuditMachine inv:

(Operation^On and Operation^Anticlockwise) @now-D

and not WithinP((Operation^Clockwise or Operation^Off),D)

and $\mathrm{D}>=\mathrm{CT}+\mathrm{MD}$ and not WithinP (State^Bottom, D)

implies WithinF(Alarm^StopWarning, 1)

The OTL specifications should be carefully studied in order to compose correctly. For instance, the statement above says that under some conditions the stop warning is issued, but it does not exclude that the stop warning is issued under other circumstances too, or even for no reason at all. The latter case should of course be excluded.

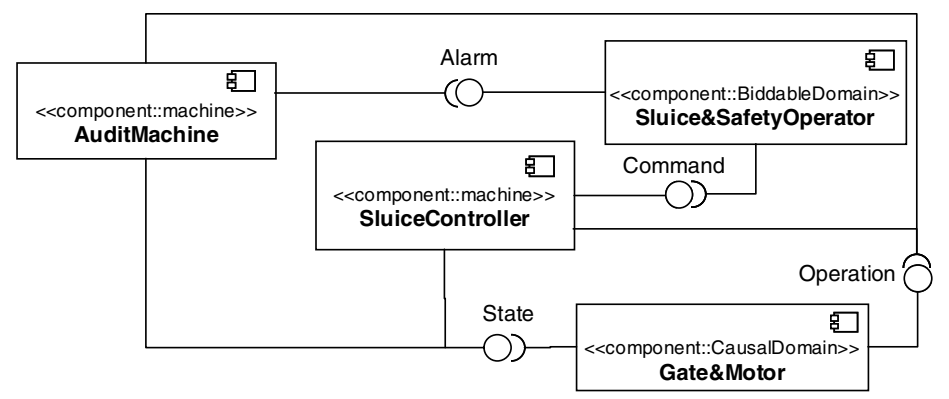

Fig. 8. Sluice gate auditing: component diagram with the Gate\&Motor\&Controller domain decomposed into its subdomains

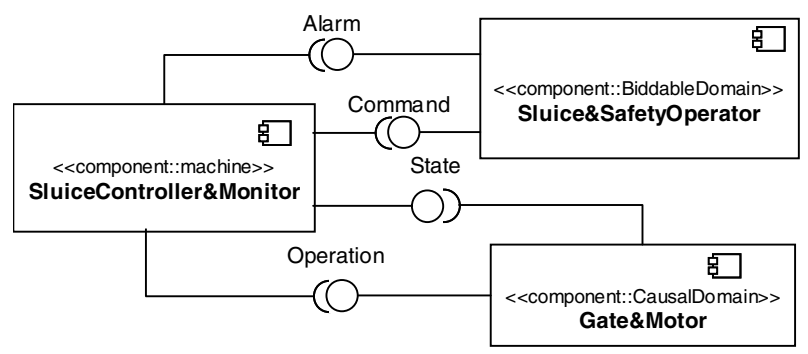

Fig. 9. Sluice gate control and auditing: the composite component diagram 
Fig. 8 represents the same diagram as Fig. 7, but with the Gate\&Motor\&Controller domain explicitly decomposed into its sub-components, which belong to the "main" PF reported in Fig. 4 and Fig. 5. It is now possible to note that the diagram in Fig. 8 includes two machines that share the same input and output signals: this suggests that the two machines can actually be merged into a single one, as is normal (and actually required in PF diagrams). The composition of the two subproblems concerning the sluice gate is reported in Fig. 9: this is the complete system that takes into account both functional and reliability requirements.

\subsection{The Identity Concern}

An identity concern arises when the machine has an interface of shared phenomena with individuals in a multiplex domain. A multiplex domain consists of multiple instances of a class that are not connected into any structure that identifies them, and that do not identify themselves [1].

This concern exists in the following problem. A patient monitoring program is required in a hospital. Each patient is monitored by an analog device which measures factors such as pulse, temperature, blood pressure, and skin resistance. The program reads these factors on a periodic basis (specified for each patient) and stores the factors in a database. For each patient, safe ranges for each factor are also specified by medical staff. If a factor falls outside a patient's safe range, or if an analog device fails, the nurses' station is notified. A simplified version of the problem diagram for the system is reported in Fig. 10.

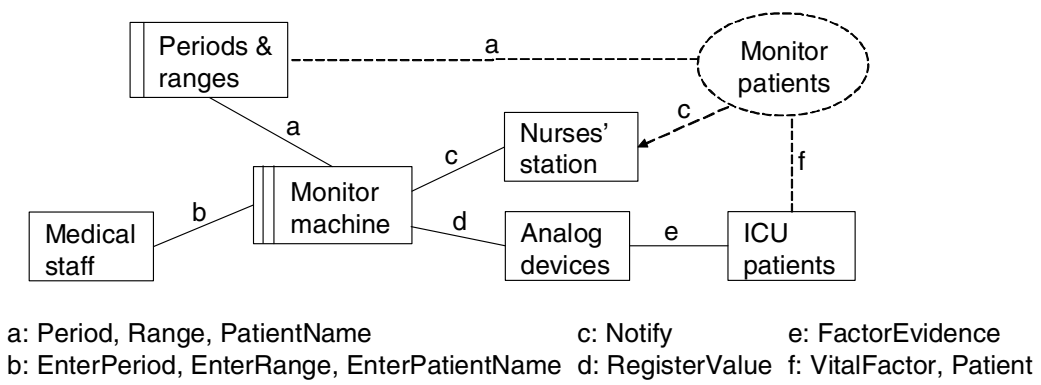

Fig. 10. Patient monitoring: partial problem diagram

Here the identity concern stems from the fact that periods and ranges are associated with patients' names, while the machine gets values that are referred to the devices (more precisely, the device and the machine share the value of a register, accessed at a machine port or storage address). It is therefore necessary to associate each patient name with the correct set of devices. This is done via an identities model domain.

The creation and maintenance of the identities model is a separate subproblem from its use. Such subproblem can be modeled as the workpieces PF reported in Fig. 11 , where the modeled reality is explicitly represented. 


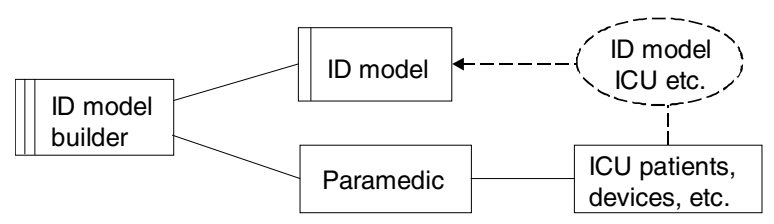

Fig. 11. Identities model creation: problem diagram

For space reasons we do not report here the whole UML model of the patient monitoring system. Instead, we illustrate the parts that are more relevant with respect to the identities concern. Fig. 12 reports the collaboration diagram of the identity modeling subproblem. Fig. 13 reports a simplified version of the class diagram of the patient monitoring system: it is possible to see that the IDmodel component includes a set of Mapping instances, each one representing a triple <Patient, Device, Register>.

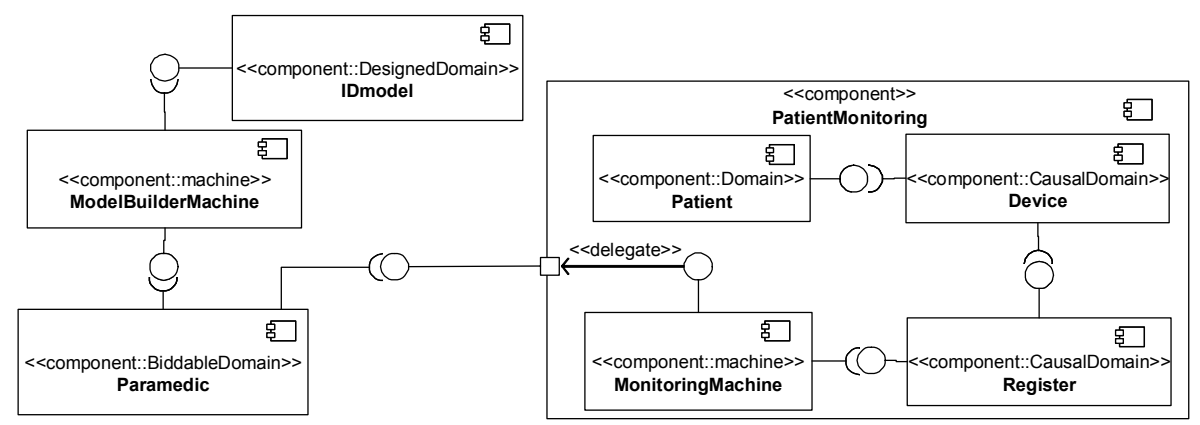

Fig. 12. Identities model collaboration diagram

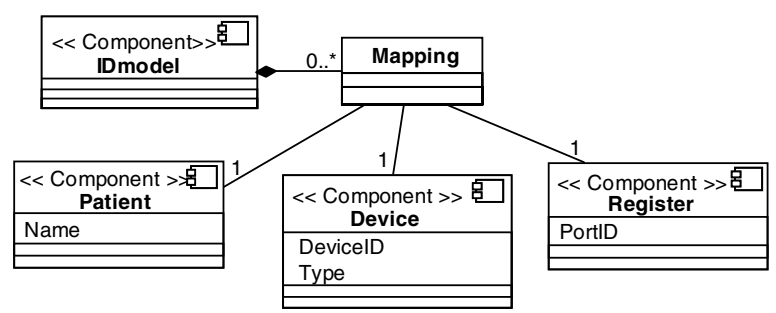

Fig. 13. Identities model: partial class diagram

Because of the simplification of the diagrams above we cannot write syntactically accurate OTL statements. Nevertheless it is easy to see that with OTL we could specify rules like the following: "whenever a patient $P$ is attached to a device $D$ which is connected to the machine $M$ via register $R$, the IDmodel must contain a mapping instance associated with P, D and R, and Rates\&Values for P must be available".

Once again, the approach based on the usage of UML and OTL proves adequate to model the considered concern in a quite straightforward way. 


\section{Related Work}

Problem frames have attracted a lot of attention from the researchers. As a result, a relevant amount of work has been done, addressing several aspects of problem frames. A first class of contributions concerns the formalization of problem frames. An early attempt to formally characterize the concepts of problem frames can be found in [12]. In [13] some problem frames are formally specified in the CASL and CASL-LTL specification languages.

A more recent work [14] provides a framework for understanding Problem Frames by locating them within the reference model for requirements engineering [15]. The semantics of problem diagrams is given in terms of "challenges". The semantics supports the textual representation of the diagrams in which Problem Frames capture problems and their relationship to solutions. This work could provide the basis for building graphical tools supporting requirement modeling through Problem Frames.

Since the development process often involves iterative, incremental definition of the problem and solution structures, the need arises to consider architectural structures, services and artifacts as part of the problem domain. Problem frames were extended in this direction, thus permitting an architecture-based approach to software development [2].

A second class of research work considers the usage of PF concepts in conjunction with UML. Konrad and Cheng [11] present a template to describe requirements patterns for embedded systems. Their approach puts together UML and problem frames, without actually merging them properly: they use the PFs to explain the patterns, while UML (class, use case and sequence diagrams) is exploited to illustrate the pattern definition.

Choppy and Reggio proposed UML-based modeling of problem frames [8]. They use plain UML to model the problem domain and the requirements: classes represent domains, while shared phenomena are modeled by means of interfaces. The behavior of the controlled domain is modeled by means of state diagrams and OCL. Requirements are expressed by means of use case diagrams, which are detailed by means of use case descriptions, including statecharts. The modeling approach proposed in [8] is adherent to the UML standard, but is less flexible then ours: because of the limitations of OCL -namely, there is no explicit notion of time- statecharts have to be employed quite extensively. This means that an operational style is enforced, and cases can arise that are difficult to model. The proposal by Choppy and Reggio is actually oriented to the definition of a method for guiding the design phase on the basis of the UML model of the problem frame. For this purpose they provide a family of patterns for the machine design, each presented by a schematic UML model.

\section{Conclusions}

The goal of the work described in this paper was to experiment with UML-oriented ways of representing problems frames, so that PF-based requirements engineering practices can be effectively integrated into the UML development process.

We showed that problem frames can be actually described by means of UML diagrams complemented with declarative specifications exploiting the OTL language. 
A first evaluation of the proposed notation performed on the basis of the examples described in Sections 2 and 3, as well as on additional examples not reported here for space reasons, lets us report the following observations:

- We found no feature of a problem domain, shared phenomenon, behavior specification, etc. that could not be expressed in the proposed UML-based notation. The UML-based notation can be used to document the requirements and specification for an information problem as done with Kovitz's checklists [7]. However, the UML-based notation seems to be more expressive, and to enable a more natural and readable style. For instance, in the sluice control system it is quite natural to represent separately the motor and the gate, to describe the motor in terms of a class with its own properties (attributes and methods), and to map methods onto interface operations, thus contributing to explain the structure and behavior of the controlled domain.

- The UML-based notation can be supported by tools. UML 2.0 compliant tools are currently being released, which support the features of UML that are relevant for our approach. Unfortunately there is no tool support for OTL, however, considering that OTL requires only a small enhancement of the OCL metamodel, it is possible that in the future some OCL tool will be extended to support OTL.

- The UML-based notation favors traceability. With our approach the notation used to describe the problem domain and the requirements is the same used to describe the design. This homogeneity makes it easier to establish/recognize dependency relations, since most relations link elements of the same nature (components, classes, attributes, states, etc.) in requirements and in design. Moreover, several tools for requirements management can import UML models, thus permitting to establish and maintain traceability relationships.

- Describing the requirements with UML makes it possible to define UML-based techniques that guide the transition from the requirements modeling phase to the design phase. An initial experimentation with such technique is reported in [8].

We consider the benefits listed above sufficiently relevant to make the proposed approach appealing in several circumstances. The work presented here can be regarded as a first step towards the definition of a methodology to employ problem frames in a UML-based development process.

\section{References}

1. Jackson, M., Problem Frames - analysing and structuring software development problems, Addison-Wesley ACM Press, 2001.

2. Hall, J.G., M. Jackson, R.C. Laney, B. Nuseibeh, and L. Rapanotti, "Relating Software Requirements and Architectures using Problem Frames", Proc. of RE02, Essen, September 2002.

3. Lavazza, L., S. Morasca, and A. Morzenti, "A Dual Language Approach to the Development of Time-Critical Systems with UML" Proc. of TACoS (Int. Workshop on Test and Analysis of Component Based Systems), Barcelona, March 27-28, 2004.

4. OMG, Unified Modeling Language: Superstructure v. 2.0, formal/05-07-04, August 2005. 
5. Lavazza, L. "Rigorous Description of Software Requirements with UML", 15th Int. Conf. on Software Engineering and Knowledge Engineering (SEKE2003), San Francisco, July 2003.

6. Heaven, W., and A. Finkelstein. "A UML Profile to Support Requirements Engineering with KAOS". IEE Proceedings - Software , vol 151, no 1, February 2004.

7. Kovitz B., Practical Software Requirements, Manning Publications, 1999.

8. Choppy, C. and Reggio, G., "A UML-Based Method for the Commanded Behavior Frame", 1st International Workshop on Advances and Applications of Problem Frames, co-located with 26th ICSE, Edinburgh, May 2004.

9. Lavazza, L. and Del Bianco, V., "A UML-based Approach for Representing Problem Frames", 1st International Workshop on Advances and Applications of Problem Frame, co-located with 26th ICSE, Edinburgh, May 2004.

10. Selic, B., Gullekson, G., Ward, P. T.: Real-Time Object-Oriented Modeling. Wiley (1994).

11. Konrad, S., Cheng, B., Requirements Patterns for Embebed Systems. IEEE Joint International Conference on Requirements Engineering, Essen, Germany, 2002.

12. Bjørner, D., Koussoube, S., Noussi, R., and Satchok, G. "Michael Jackson's Problem Frames: Towards Methodological Principles of Selecting and Applying Formal Software Development Techniques and Tools". 1st IEEE International Conference on Formal Engineering Methods, Hiroshima, 1997.

13. Choppy, C. and Reggio, G., "Using CASL to Specify the Requirements and the Design: A Problem Specific Approach." In D. Bert and C. Choppy (eds.) Recent Trends in Algebraic Development Techniques, Selected Papers of WADT'99, LNCS 1827, Springer Verlag, 2000.

14. Hall, J., Rapanotti, L., and Jackson, M. "Problem frame semantics for software development”, Software and Systems Modeling, vol. 4, n. 2, Springer-Verlag, July 2005.

15. Gunter C.A., Gunter E.L., Jackson M., and Zave P., "A Reference Model for Requirements and Specifications", IEEE Software, vol. 17, n. 3, May-June 2000.

16. Jacobson, I., "Object-Oriented Development In an Industrial Environment", Proc. of OOPSLA '87.

17. Glinz, M., "Problems and Deficiencies of UML as a Requirements Specification Language", Proc. of the $10^{\text {th }}$ IWSPD, San Diego, November 2000.

18. Hurlbut, R.R., "A Survey of Approaches For Describing and Formalizing Use Cases", http://www.iit.edu/ rhurlbut/xpt-tr-97-03.html.

19. OMG, “OCL 2.0 Specification V. 2.0”, ptc/2005-06-06, June 2005. 